\title{
Efeitos da craniopuntura de Yamamoto na osteoartrite de joelho: estudo de caso
}

\author{
The Yamamoto scalp acupuncture effects in knee osteoarthritis: a case study
}

Lorine da Silva ${ }^{1}$, Alda Nerys Taxoto ${ }^{1}$, Elaine Meque Montalvão ${ }^{2}$, Amélia Pasqual Marques ${ }^{3}$, Patrícia Pereira Alfredo ${ }^{4}$

Estudo desenvolvido no Ambulatório de Acupuntura do Centro de Aprimoramento em Saúde (CAPRIS) - Osasco (SP), Brasil.

${ }^{1}$ Fisioterapeuta especialista em Acupuntura.

2 Professora especialista do Curso de Acupuntura do CAPRIS Osasco (SP), Brasil.

${ }^{3}$ Professora Doutora do Curso de Fisioterapia - Departamento de Fonoaudiologia, Fisioterapia e Terapia Ocupacional da Universidade de São Paulo (USP) - São Paulo (SP), Brasil.

${ }^{4}$ Professora Mestre do Curso de Acupuntura do CAPRIS Osasco (SP), Brasil.

ENDEREÇO PARA CORRESPONDÊNCIA

Patrícia Pereira Alfredo - Av. Profa. Gioconda Mussolini, 291 - apto 13 - Jardim Risso - CEP: 05587-120 - São Paulo (SP), Brasil. - E-mail: patriciaalfredo@yahoo.com.br

\section{APRESENTACÃO}

maio. 2010

ACEITO PARA PUBLICAÇÃO jan. 2011

FONTE DE FINANCIAMENTO nenhuma

CONFLITO DE INTERESSES nada a declarar
RESUM0: O objetivo deste estudo foi verificar os efeitos da craniopuntura de Yamamoto na dor, na amplitude de movimento, qualidade de vida e funcionalidade de paciente com osteoartrite (OA) de joelho. Foi realizado um estudo de caso de uma paciente de 59 anos apresentando quadro de dor no joelho, redução funcional nos últimos três meses e radiografia constando o grau 3 de OA de joelhos. A avaliação foi feita antes e após o tratamento através da Escala Visual Analógica (EVA) para avaliação da dor, da qualidade de vida pelo questionário Western Ontario and McMaster Úniversities Osteoarthritis (WOMAC), da funcionalidade pelo Knee injury and Osteoarthritis Outcome Score (KOOS) e da amplitude de movimento do joelho pela goniometria. A craniopuntura foi realizada duas vezes por semana, com duração de 40 minutos cada, totalizando 10 sessões. A análise dos dados foi feita de forma descritiva. A variável utilizada foi o ganho relativo (GR) medido no pré e no pós-tratamento. A dor no joelho diminuiu ( $G R=100 \%)$, enquanto a amplitude de movimento aumentou em ambos os joelhos (GR=20\%). Observou-se melhora na dor ( $G R=83 \%$ ), na rigidez $(\mathrm{GR}=50 \%)$, na função $(\mathrm{GR}=80 \%)$ e no escore total da WOMAC $(\mathrm{GR}=79 \%)$. Verificou-se, através da KOOS, melhora nos sintomas (GR=8\%), na dor (GR=26\%), nas atividades de vida diária $(G R=21 \%)$ e na qualidade de vida $(G R=17 \%)$. Concluise que a craniopuntura foi efetiva no alívio da dor, na amplitude de movimento, qualidade de vida e funcionalidade de paciente com OA de joelho.

DESCRITORES: acupuntura; osteoartrite; dor; joelho.

ABSTRACT: The purpose of this study was to investigate the effects of Yamamoto New Scalp Acupuncture in pain, range of motion, life quality and functionality of patients with knee osteoarthritis (OA). We conducted a case study of a patient of 59 years complaining of knee pain, functional reduction in the last three months and radiography consisting of grade 3 knee OA. The evaluation was done before and after treatment using the Visual Analogue Scale index (VAS) for pain assessment, life quality by Western Ontario and McMaster Universities Osteoarthritis index (WOMAC), the functionality for Knee injury and Osteoarthritis Outcome Score index (KOOS) and range of motion of the knee by goniometry. The craniopuncture was performed twice on week, lasting 40 minutes, totaling 10 sessions. Data analysis was performed descriptively. The variable used was the relative gain (GR) measured before and after treatment. The knee pain decreases $(G R=100 \%)$, while the range of motion increases in both knees (GR=20\%). An improvement in pain (GR=83\%), stiffness $(G R=50 \%)$, function ( $G R=80 \%)$ and total score of WOMAC (GR=79\%). By KOOS, it was found, improvement in symptoms $(G R=8 \%)$, pain $(G R=26 \%)$ in activity od daily living $(G R=21 \%)$ and life quality $(G R=17 \%)$. We conclude that the craniopuncture was effective in pain relieving, motion range, life quality and functionality of patient with knee osteoarthritis.

KEYWORDS: acupuncture; osteoarthritis; pain; knee. 


\section{INTRODUCÃO}

Enfermidades degenerativas são consideradas como problemas de saúde pública, uma vez que desencadeiam um elevado número de aposentadorias por invalidez. Dentre essas enfermidades está a osteoartrose (OA), uma doença degenerativa que acomete as articulações, com causa desconhecida e multifatorial, sendo que em alguns casos aparece como consequência de outra doença óssea ou articular que predisponha a deterioração'.

A OA é a doença reumática mais prevalente, afetando cerca de $10 \%$ da população ocidental. No Brasil, é responsável por $7,5 \%$ de todos os afastamentos do trabalho e 6,2\% das aposentadorias ${ }^{2}$. Era vista anteriormente como uma doença de avanço gradual, com limitadas possibilidades de intervenção terapêutica, mas na atualidade, pode ser modificada quando tratada precocemente e com acompanhamento a longo prazo ${ }^{3}$.

Dentre as formas clínicas de OA, destacam-se a de quadril e de joelho por serem particularmente mais incapacitantes ${ }^{4}$.

A queixa de dor no joelho é muito frequente e, em geral, é provocada pelo desequilíbrio muscular decorrente do envelhecimento e do processo degenerativo, tão comum nesses pacientes. $\mathrm{O}$ desequilíbrio muscular caracteriza-se por atrofia do quadríceps associada à retração dos músculos posteriores de coxa, o que leva ao posicionamento em flexão do joelho. A dor decorrente desse processo degenerativo tem característica progressiva e evolutiva, piorando com a atividade física ${ }^{5}$.

O quadro doloroso e as alterações secundárias musculares, tendinosas e ligamentares são as maiores responsáveis pela incapacidade funcional do paciente ${ }^{6}$.

Os principais objetivos no tratamento da OA são: reduzir os sintomas, manter ou aumentar a amplitude de movimento (ADM), minimizar as perdas funcionais e limitar a progressão das alterações estruturais, visando impedir a diminuição da qualidade de vida dos pacientes ${ }^{7}$.

A acupuntura é uma das terapias mais antigas e eficazes de tratamento, praticada na China durante os últimos 5 mil anos; visa o equilíbrio "energético" do organismo por meio de estímulos nos acupontos, ao longo de trajetos energéticos localizados na epiderme, conhecidas como meridianos. Alguns de seus mecanismos de ação, como a analgesia são comprovados pela medicina ocidental ${ }^{8}$.

A craniopuntura de Yamamoto $^{9}$ é uma das técnicas da acupuntura que usa métodos de punção tradicionais de acupuntura, em combinação com o conhecimento ocidental de anatomia, fisiologia, patologia e neurologia, sobre as partes relacionadas do couro cabeludo que correspondem ao córtex cerebral. Segundo Yamamoto e Yamamoto ${ }^{9}$, esta técnica não apresenta efeitos colaterais e pode reduzir drasticamente o consumo de medicamentos.

Apesar da craniopuntura ser uma técnica rápida e de fácil aplicação, não há estudos sobre sua aplicação e efeitos na dor provocada pela OA de joelho. Sendo assim, este trabalho teve como objetivo verificar os efeitos da craniopuntura de Yamamoto na dor, na amplitude de movimento, qualidade de vida e funcionalidade de pacientes com OA de joelho.

\section{METODOLOGIA}

Trata-se de estudo de caso. Foi selecionada uma paciente de 59 anos de idade, sexo feminino, apresentando OA de joelho, recrutada no ambulatório de acupuntura do Centro de Aprimoramento em Saúde - Osasco (SP).

Os critérios de inclusão foram: apresentar quadro de dor no joelho, redução funcional nos últimos três meses e radiografia constando o grau 2-4 de $\mathrm{OA}^{10}$.

Foram excluídos indivíduos portadores de distúrbios mentais, problemas neurológicos, diabetes, deficiência visual ou auditiva, outras lesões na articulação do joelho (ligamentares, meniscais ou musculares) não inerentes à OA, prótese total ou parcial, fibromialgia, artrite reumatóide, lúpus eritematoso sistêmico, doenças reumáticas sistêmicas e a realização de outro tratamento e uso de fármacos (analgésicos) nos últimos três meses.

A pesquisa foi aprovada pelo Comitê de Ética em Pesquisa do Centro Regional Universitário do Espírito Santo do Pinhal (UNIPINHAL) e a participante assinou um termo de consentimento livre e esclarecido. Toda intervenção foi realizada no ambulatório de acupuntura do Centro de Aprimoramento em Saúde e as avaliações foram realizadas por um avaliador cego, o mesmo era fisioterapeuta e foi treinado para execução das avaliações e instruído a não relatar antes do término do estudo nenhum dos dados encontrados ao fisioterapeuta executante do protocolo de tratamento.

Avaliação

A paciente foi avaliada antes e após o tratamento com os seguintes recursos: anamnese, avaliação da dor pela Escala Visual Analógica (EVA), goniometria do joelho através de Goniômetro Universal, avaliação da funcionalidade pelo questionário Knee Injury and Osteoarthritis Outcome Score $(\mathrm{KOOS})^{11}$ e da qualidade de vida pelo questionário Western Ontario and McMaster Universities Osteoarthritis (WOMAC) ${ }^{12}$.

$\mathrm{Na}$ anamnese, foram colhidos os dados pessoais, peso, altura e foi feita uma investigação sobre doenças pré-existentes (diabetes melitus, alterações da tireóide, colesterol, hipertensão arterial). Também foram verificados os dados vitais (pressão arterial, frequência cardíaca e frequência respiratória).

Avaliação da dor: uma escala visual analógica foi usada para registrar a intensidade da dor nos joelhos acometidos. A paciente foi instruída a marcar com uma caneta em uma linha de $10 \mathrm{~cm}$, a intensidade da dor aos movimentos. O zero indicava sem dor e 10 a pior dor imaginável ${ }^{13}$.

Goniometria: foi avaliada a amplitude de movimento dos joelhos com o goniômetro manual ${ }^{14}$.

Avaliação da funcionalidade: o questionário KOOS ${ }^{11}$ foi utilizado. É autoaplicável, composto por 42 questões que avaliam 5 variáveis: dor, sintomas, atividades de vida diária (AVDs), desporto e atividades de recreação, e qualidade de vida. Quanto maior o escore, maior o comprometimento.

Avaliação da qualidade de vida: foi utilizado o questionário WOMAC ${ }^{12}$. É um instrumento de autoavaliação da qualidade de vida (dor, rigidez articular e atividade física). Quanto maior o escore, maior é o impacto da OA na qualidade de vida.

\section{Intervenção}

A craniopuntura foi administrada com a paciente em decúbito dorsal, através da 
aplicação de agulhas de acupuntura de comprimento médio $n^{\circ} 5(0,25 \times 40 \mathrm{~mm})$, esterilizadas, descartáveis e de aço inoxidável (Dong Bang) nos pontos G1, G2 e G3 (Figura 1), indicados para gonartrose, localizados na área temporal de implantação dos cabelos e mantidas por 40 minutos.

Foram realizadas duas sessões semanais de 40 minutos, totalizando 10 sessões.

\section{Análise dos dados}

Por se tratar de um estudo de caso, os dados foram analisados de forma descritiva. A variável utilizada foi o ganho relativo (GR) medido no pré e no pós-tratamento.

Considera-se GR=100*(pós-pré)/pré no caso onde aumento significa melhora ou $\mathrm{GR}=100 *$ (pré-pós)/pré no caso onde diminuição representa melhora.

\section{RESULTADOS}

A Tabela 1 mostra que a dor no joelho diminuiu (GR=100\%), enquanto a amplitude de movimento aumentou em ambos os joelhos $(\mathrm{GR}=20 \%)$. Os dados da qualidade de vida foram avaliados com o WOMAC antes e após o tratamento. Nestes, houve melhora na dor (GR=83\%), na rigidez (GR=50\%), na função ( $G R=80 \%$ ) e no escore total $(G R=79 \%)$ antes e após o tratamento. Os dados da dor, sintomas, AVDs, recreação e qualidade de vida, foram avaliados com o KOOS. Nestes, houve melhora nos sintomas ( $G R=8 \%$ ), na dor $(G R=26 \%)$, nas atividades de vida diária $(G R=21 \%)$ e na qualidade de vida (GR=17\%). Não houve melhora nas atividades de recreação $(\mathrm{GR}=0 \%)$.

\section{DISCUSSÃO}

O objetivo deste estudo foi verificar a eficácia da craniopuntura de Yamamoto na dor, na amplitude de movimento, qualidade de vida, sintomas e AVDs de paciente com OA de joelho. Os resultados obtidos mostram que a craniopuntura foi efetiva no alívio da dor, na amplitude de movimento, qualidade de vida e funcionalidade.

Vários estudos ${ }^{15-18}$ apontaram a eficácia da acupuntura no alívio da dor no tratamento de OA de joelho. Bjordal et al. ${ }^{19}$ avaliaram o efeito analgésico do

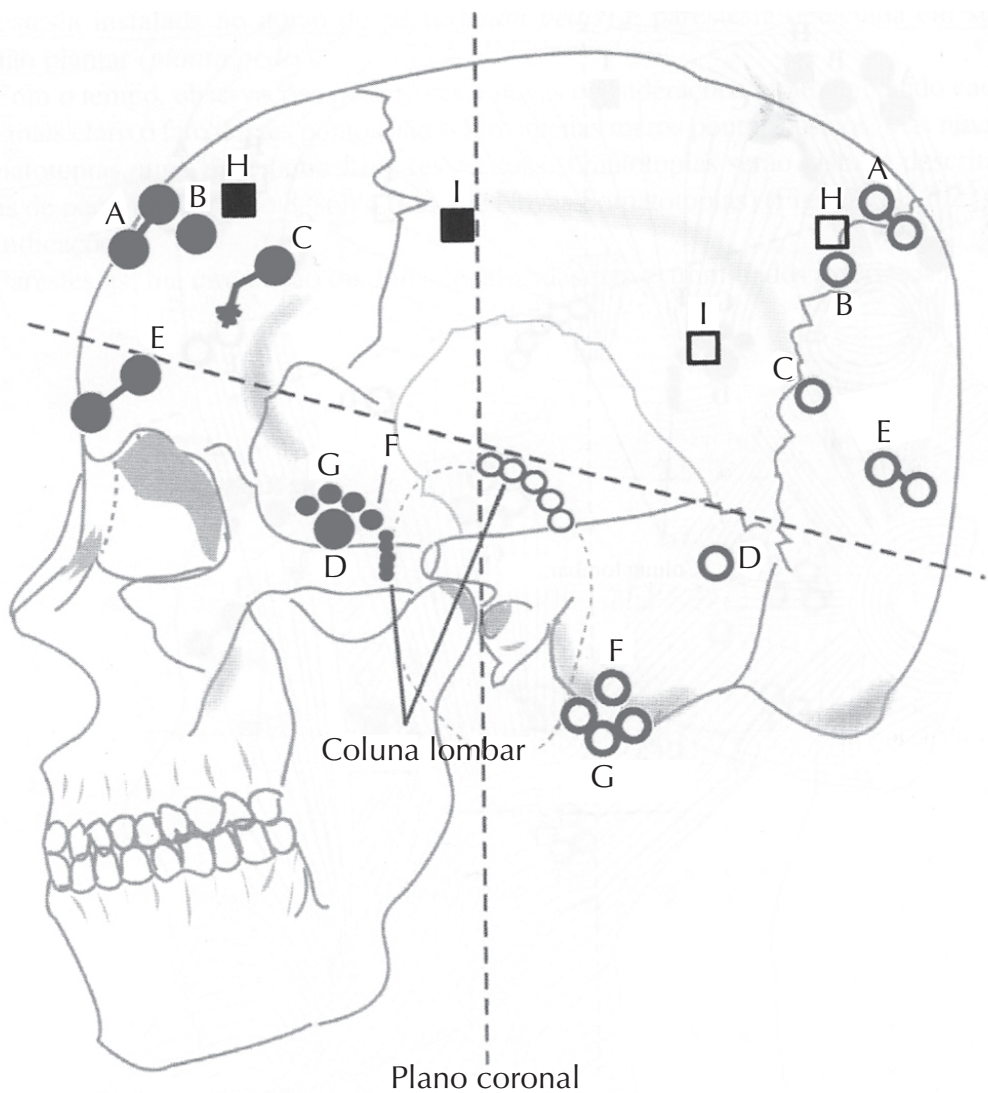

Fonte: Yamamoto, Schockert e Boroojerdi ${ }^{24}, 2007$

Figura 1. Pontos básicos da nova craniopuntura de Yamamoto sobre o crânio, localizações Yin e Yang

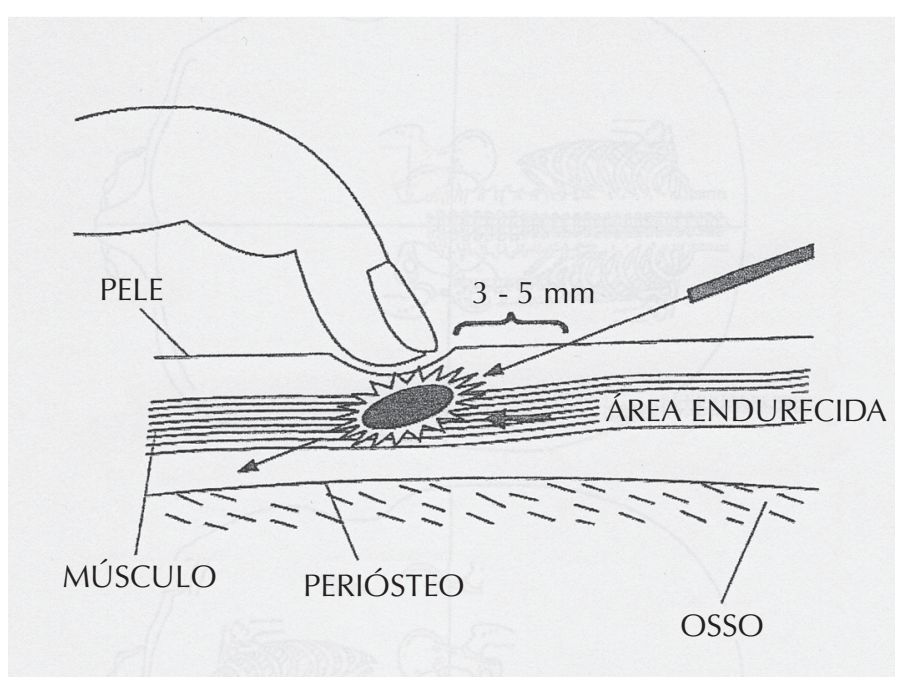

Fonte: Boucinhas ${ }^{26}, 2002$

Figura 2. Técnica de inserção das agulhas

laser de baixa potência (LBP), da estimulação elétrica transcutânea (TENS) e da eletro-acupuntura em pacientes com OA de joelho, durante um tratamento de quatro semanas e constataram que todos os tratamentos foram eficazes no alívio da dor. No presente estudo, a craniopuntura também se mostrou eficaz no alívio da dor de paciente com OA de joelho.

Já Ezzo et al. ${ }^{21}$ em outra revisão sistemática, constataram que há fortes evidências de que a aplicação da acupuntura para o alívio da dor é mais 
Tabela 1. Resultados para dor e amplitude de movimento, dados da qualidade de vida avaliada com o questionario Western Ontario and McMaster Universities Osteoarthritis (WOMAC), dados da dor, sintomas, atividades de vida diária, recreação e qualidade de vida avaliados com o questionário Knee Injury and Osteoarthritis Outcome Score (KOOS)

\begin{tabular}{|c|c|c|c|}
\hline Variáveis & Antes & Após & $\mathrm{GR}(\%)$ \\
\hline Dor $(\mathrm{cm})^{*}$ & 5 & 0 & 100 \\
\hline \multicolumn{4}{|l|}{ ADM (graus) } \\
\hline Joelho direito & 100 & 120 & 20 \\
\hline Joelho esquerdo & 100 & 120 & 20 \\
\hline \multicolumn{4}{|l|}{ WOOMAC } \\
\hline Dor $(0-20)$ & 6 & 1 & 83 \\
\hline Rigidez (0-8) & 2 & 1 & 50 \\
\hline Função (0-68) & 20 & 4 & 80 \\
\hline Escore total & 28 & 6 & 79 \\
\hline \multicolumn{4}{|l|}{ KOOS (0-100) } \\
\hline Sintomas & 89,3 & 96,4 & 8 \\
\hline Dor & 75,0 & 94,4 & 26 \\
\hline AVD & 77,9 & 94,1 & 21 \\
\hline ADL & 70,0 & 70,0 & 0 \\
\hline QL & 37,5 & 43,7 & 17 \\
\hline
\end{tabular}

GR: ganho relativo; ADM: amplitude de movimento; AVD: atividade de vida diária; ADL: atividades de desporto e recreação; QL: qualidade de vida; *escala visual analógica de dor (EVA)

efetiva quando comparada ao placebo, mas que para a função do joelho, existem limitadas evidências sobre uma maior eficácia da aplicação da acupuntura em relação ao placebo. No nosso estudo de caso, com a aplicação da técnica de craniopuntura de Yamamoto, foi observada além da melhora da dor, uma melhora funcional na paciente com OA.

As intervenções farmacológicas são crescentes no tratamento da OA visando o alívio da dor. Segundo Ghosh ${ }^{22}$, os medicamentos comumente utilizados para o alívio da dor e da inflamação de pacientes com OA, podem similarmente inibir os mecanismos de reparação da cartilagem.
Bjordal et al. ${ }^{23}$, em uma metanálise sobre os efeitos dos anti-inflamatórios não esteroidais (NSAIDs) no alívio da dor de pacientes com OA de joelho perceberam que mesmo que ocorra uma diminuição do quadro doloroso, seu uso prolongado pode causar aumento da pressão sanguínea, falência renal, entre outros. Concluindo assim que sua utilização deve ocorrer por um tempo limitado.

Yamamoto Schockert e Boroojerdi ${ }^{24}$ relataram que a craniopuntura é uma técnica utilizada também para o alívio da dor, que não apresenta efeitos colaterais e pode reduzir drasticamente o consumo de medicamentos. Fato comprovado no presente estudo, onde a paciente não precisou fazer uso de medicamento para analgesia durante a intervenção e não apresentou efeitos colaterais com a aplicação da técnica.

Vas et al. ${ }^{25}$ analizaram a eficácia da acupuntura como terapia complementar ao tratamento farmacológico da OA de joelho no que diz respeito ao alívio da dor mensurada através da EVA, e, redução da rigidez articular, funcionalidade, qualidade de vida mensuradas através do WOMAC e no consumo de diclofenaco durante o tratamento. Observaram que a acupuntura, como terapia complementar ao tratamento farmacológico, é mais efetiva que o tratamento farmacológico administrado isoladamente na redução da dor, rigidez, funcionalidade e qualidade de vida. No presente estudo de caso, a acupuntura foi aplicada isoladamente e foi observada uma melhora em todas essas variáveis.

A craniopuntura é uma técnica de tratamento simples e que surte efeitos relativamente interessantes. Vale ressaltar que este é um estudo de caso e que apenas um paciente não é suficiente para determinar a eficácia de qualquer modalidade terapêutica. Sugere-se que novas investigações sejam realizadas com um número maior de pacientes, para fundamentar melhor a eficácia da técnica.

\section{CONCLUSÃO}

A craniopuntura de Yamamoto foi efetiva no alívio da dor, na amplitude de movimento, qualidade de vida e funcionalidade de paciente com OA de joelho.

\section{REFERÊNCIAS}

1. Arrebola AP. Papel del ejercicio físico en el paciente com artrosis. Rehabilitación. 2003;37(6):307-22.

2. Chachade WH, Giorgi RDN, Pastor EMH. Osteoartrose. Rev Bras Med. 2001;58(5):304-14.

3. Jamtvedt G, Dahm KT, Christie A, Moe RH, Haavardsholm E, Holm I, et al. Physical therapy interventions for patients with osteoarthritis of the knee: an overview of systematic reviews. Phys Ther. 2008;88(1):123-36.
4. Ricci NA, Coimbra IB. Exercício físico como tratamento na osteoartrite de quadril: uma revisão de ensaios clínicos aleatórios controlados. Rev Bras Reumatol. 2006;46(4):273-80.

5. Camanho GL. Dor aguda no joelho do paciente idoso. Rev Bras Ortop. 2008;43(9):361-6.

6. Greve JMD, Plapler PG, Seguchi HH, Pastor EH, Baptistella LR. Tratamento fisiátrico da dor na osteoartose. Rev Hosp Clin Fac Med. 1992;47(4):185-9. 


\section{Referências (cont.)}

7. Pelletier JP, Martel-Pelletier J, Abramson SB. Osteoarthritis, an inflammatory disease: potential implication for the selection of new therapeutic targets. Arthritis Rheum. 2001;44(6):1237-47.

8. Wang SM, Kain ZN, White P. Acupuncture analgesia: I: The scientific basis. Anesth Analg. 2008;106(2):602-10.

9. Yamamoto T, Yamamoto H. Yamamoto New Scalp Acupuncture "YNSA". Tokyo: Axel Springer Japan Publishing Inc, 1998.

10. Kellgren, JH, Lawrence, JS. (1957) Radiological assessment of osteo-arthrosis. Ann Rheum Dis. 1957;16(4):494-502.

11. Ross EM, Toksvig-Larsen S. Knee injury and Osteoarthritis Outcome Score (KOOS): rom joint injury to osteoarthritis. Health Qual Life Outcomes. 2003; 1:64.

12. Bellamy N, Buchnan WW, Goldsmith $\mathrm{CH}$, Campbell J, Stitt LW. Validation study of WOMAC: a health status instrument for measuring clinically important patient relevant outcomes to antirheumatic drug therapy in patients with osteoarthritis of the hip or knee. J Rheumatol. 1988;15(12):1833-40.

13. Huskisson EC. Measurement of pain. Lancet. 1974;2(7889):1127-31.

14. Marques AP. Manual de goniometria. 2a ed. São Paulo: Manole, 2003.

15. Witt C, Brinkhaus B, Jena S, Linde K, Streng A, Wagenpfeil $S$, et al. Acupuncture in patients with osteoarthritis of the knee: a randomised trial. Lancet. 2005;366(9480):136-43.

16. Berman BM, Singh BB, Lao L, Langenberg P, Li H, Hadhazy $\mathrm{V}$, et al. A randomized trial of acupuncture as an adjunctive therapy in osteoarthritis of the knee. Rheumatology (Oxford). 1999;38(4):346-54.

17. Tukmachi E, Jubb R, Dempsey E, Jones P. The effects of acupuncture on the symptoms of knee osteoarthritis an open randomised controlled trial. Acupunct Méd. 2004;22(1):14-22.
18. Vas J, Méndez C, Perea-Milla E, Vega E, Panadero MD, Leon, JM, et al. Acupuncture as a complementary therapy to the pharmacological treatment of osteoarthritis of the knee; randomised controlled trial. Br Med J. 2004;329:1216-9.

19. Bjordal JM, Johnson MI, Martins LR, Bogen B, Chow R, Ljunggren AE. Short-term efficacy of physical interventions in osteoarthritic knee pain. A systematic review and meta-analysis of randomised placebocontrolled trials. BMC Musculoskelet Disord. 2007;8:51.

20. Ernst E. Acupuncture as a Symptomatic Treatment of Osteoarthritis: A systematic review. Scand J Rheumatol. 1997;26:444-7.

21. Ezzo J, Hadhazy V, Birch S, Lao L, Kaplan G, Hochberg $M$, et al. Acupuncture for Osteoarthritis of the Knee: A systematic review. Arthritis Rheum. 2001;44(4):819-25.

22. Ghosh P. Articular cartilage: what it is, why it fails in osteoarthritis, and what can be done about it. Arthritis Care Res. 1989;4:211-20.

23. Bjordal JM, Ljunggren AE, Klovning A, Slordal L. Nonsteroidal anti-inflammatory drugs, including cyclooxygenase-2 inhibitors, in osteoarthritic knee pain: meta-analysis of randomized placebo controlled trials. BMJ. 2004;329(7478):1317.

24. Yamamoto T, Schockert T, Boroojerdi B. Treatment of juveline stroke using Yamamoto New Scalp Acupuncture (YNSA) - a case report. Acupunct Med. 2007;25(4):200-2.

25. Vas J, Méndez C, Milla EM, Vega E, Panadero MD, León $\mathrm{JM}$, et al. Acupuncture as a complementary therapy to the pharmacological treatment of osteoarthritis of the knee: randomised controlled trial. BMJ. 2004;329(7476):1216.

26. Boucinhas J. YNSA - A nova acupuntura craniana de Yamamoto. Apresentação do Dr. Raphael Nogier. 2 ed. revista e modificada. Natal: SMB; 2002. 